\title{
Attitudes towards individuals with halitosis: an online cross sectional survey of the Dutch general population
}

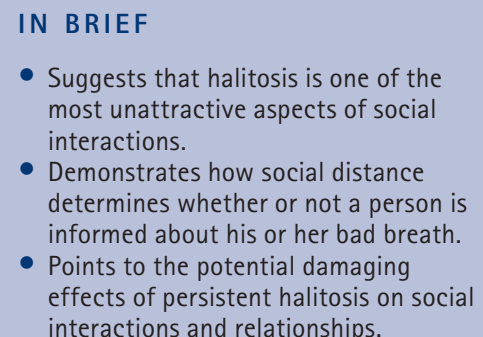

\author{
A. de Jongh, ${ }^{* 1,2}$ A. J. van Wijk, ${ }^{1}$ M. Horstman ${ }^{3}$ and C. de Baat ${ }^{4}$
}

\section{VERIFIABLE CPD PAPER}

\begin{abstract}
Objective To derive an estimate of encounters with halitosis and to assess the impact of halitosis on their psychosocial interactions with halitosis patients. Methods Participants were 1,006 members of an online panel, being representative of the population of the Netherlands with regard to gender, age, family situation, education level and labour participation. They were invited to participate in a survey using an online questionnaire with four questions on becoming faced with people having halitosis. Results Almost 90\% of the participants indicated being faced with a person having halitosis regularly, 40\% at least once a week, and men significantly more frequently than women. Halitosis was reported to be a strong 'downer' when meeting a person for the first time. Whether one would draw a person's attention to his breath malodour proved to be related to the kind of person involved and appeared to decrease with the increase of the social distance to the person. Drawing a person's attention to his breath malodour would virtually always be performed personally. Conclusion Halitosis is considered to be one of the most unattractive aspects of social interactions and has potentially damaging effects on psychosocial interactions and relationships.
\end{abstract}

Halitosis, or breath malodour, is a common problem, which is multifactorial. In $80-90 \%$ of cases it is caused by oral conditions, defined as oral malodour. Oral malodour arises from microbial degradation of organic substrates. It is estimated that about $30 \%$ of the population suffer from halitosis. ${ }^{1-5}$

Interestingly, there is almost no literature concerning potential unattractive aspects of appearance, odours and behaviour, and this applies even more to the extent to which these aspects impact on psychosocial interactions. Yet, in the case of halitosis it is conceivable that people feel insecure in contact with other persons, because they fear that these persons might smell their breath malodour, whereas those faced with halitosis may literally take distance from the person involved. Although psychosocial aspects are considered to play a role in terms of how

\footnotetext{
Section Behavioural Sciences, Academic Centre for Dentistry Amsterdam (ACTA), Amsterdam, the Netherlands; ${ }^{2}$ School of Health Sciences, Salford University, Manchester, United Kingdom; ${ }^{3}$ Marketing Research Office Kien, Groningen, the Netherlands; ${ }^{4}$ Department of Oral Function and Prosthetic Dentistry, Radboud University Nijmegen Medical Centre, Nijmegen, the Netherlands

${ }^{*}$ Correspondence to: Professor Ad de Jongh

Email: a.de.jongh@acta.nl; Tel: +31 (0) 623,715,237
}

Online article number E8

Refereed Paper - accepted 12 December 2013

DOI: $10.1038 / s j . b d j .2014 .101$

${ }^{\circ}$ British Dental Journal 2014; 216: E8 halitosis affects relationships and contact with other people. ${ }^{6-12}$ To our best knowledge only one study examined the impact of (genuine) halitosis on people's psychosocial interactions. The results of this study showed that interactional difficulties were related to characteristics of social anxiety as indexed by feelings of discomfort or fear in psychosocial interactions, involving concerns about being judged or evaluated. ${ }^{13}$ Not surprisingly, among halitosis patients with a high level of social anxiety, the majority (68.3\%) indicated that they could not talk with people. However, 48\% of halitosis patients with a relatively low level of social anxiety reported the same problem. These findings suggest that halitosis hampers psychosocial interactions at least of halitosis patients themselves, but maybe also of persons faced with halitosis patients.

The purposes of the present study were to derive an estimate of the prevalence of being faced with halitosis, and to assess the impact of halitosis on psychosocial interactions. The first aim was to assess in a representative sample of the population of the Netherlands the prevalence of encounters with halitosis. The second aim was to determine among them the impact of halitosis through comparing halitosis with other potential unattractive aspects of social interactions in terms of 'downers'. The third aim was to assess the extent to which they would draw attention to a person's halitosis. The fourth aim was to collect information about how they generally would raise the issue of halitosis when psychosocially interacting with a person having halitosis.

\section{METHODS}

This study was conducted among members of an online survey panel assembled by the Internet survey company Panelwizard (http:// www.panelwizard.com). The panel consisted of approximately 20,000 individuals aged 16 years and older, being representative for the population of the Netherlands with regard to gender, age, family situation, education level, and labour participation. The study was performed in accordance with the precepts and regulations for research as stated in the Declaration of Helsinki and the Dutch Medical Research on Humans Act (WMO) concerning scientific research. To the current type of social science research, the WMO is not applicable. Written informed consent was obtained from each study participant.

An independent, random, representative panel sample was invited to participate in a survey using an online questionnaire in the Dutch language with four questions. The number of approached panel members was 1,$663 ; 1,006(62 \%)$ agreed to participate within a previously determined time frame of four days; 1,002 completed the entire 
questionnaire. The data collected were obtained directly by the survey company, which prepared and delivered a de-identified SPSS data file to the first author.

The first question 'How often are you faced with people having breath malodour?' had five response categories: 'daily', 'weekly', 'less than weekly', 'never', and 'I do not know'. By the second question the participants were requested to compare the impact of halitosis with three other potential unattractive aspects of psychosocial interactions in terms of a 'downer'. The question and response categories were formulated as follows: 'What would be your strongest 'downer' (Dutch: 'afknapper') when meeting a person for the first time?': 'sweat malodour', 'breath malodour', 'dirty ears', and 'dishevelled appearance due to facial, ear or nasal hair'. The third question was 'Suppose one of the following persons in your environment ('your spouse', 'a family member', 'a friend', 'a colleague', 'your boss or supervisor', and 'an unknown person you would accidentally meet in a train or shop') has breath malodour, would you draw his/ her attention to this problem?' ('Yes', 'No'). The last question referred to the method of raising the problem of halitosis: 'I would not point out the problem', 'I would point out it personally', 'by letter/e-mail', 'by sms/ ping/whatsapp', 'through dentist/dental hygienist', and 'through somebody else').

\section{Statistical analysis}

The survey data were analysed using the computer programme Statistical Package for Social Sciences (SPSS) 18.0. Statistical tests included the Student's t-test (or MannWhitney U test for comparing ordinal measurements) and analysis of variance (ANOVA) for comparisons of continuous variables (self-perceived breath odour) among the various socio-demographic groups. Chi-square tests were used for associations between categorical variables. To test whether our sample is similar to the general population aged 16 years and older (with respect to the demographic variables), a chi-square test was used to compare the observed frequencies in our sample with the expected frequencies based on population data. In order to compare proportions, 95\% confidence intervals (CI) were calculated. A p-value of less than 0.05 was considered to indicate statistical significance.

\section{RESULTS}

\section{Sample characteristics}

The socio-demographic characteristics of the participants are presented in Table 1 . The distributions of these characteristics proved to be an accurate reflection of the 2011 data of the population of the Netherlands aged 16 years and older, published by the Central Bureau of Statistics (CBS). ${ }^{14}$ The frequencies of the relevant socio-demographic characteristics of the participants did not differ significantly from the general population aged 16 years and older of the Netherlands (p-values 0.19-0.70).

\section{Being faced with halitosis}

The vast majority of the participants (89.3\%) reported being regularly faced with a person having halitosis. Nearly four out of ten participants (39.8\%) indicated to face a person having halitosis at least once a week, $14.5 \%$ daily and 25.3\% weekly, $49.5 \%$ less than weekly, 6.2\% never, and 4.5\% did not know. Men were significantly more likely than women to be faced daily or weekly with a person having halitosis (44.7\% vs 35.0\%; chi-square test [4]: 10.6; $p=0.032$ ). Participants with labour participation reported to be confronted with a person having halitosis daily or weekly significantly more often than participants without labour participation (50.1\% vs 26.7\%; chi-square test [4]: 68.6; $p<0.001)$. No gender difference was found with regard to this association of labour participation and being faced with a person having halitosis daily or weekly. The results of the chi-square test for men and women separately were (4) 38.5 and (4) 26.5 respectively ( $\mathrm{p}<0.001)$.

\section{Halitosis in terms of a 'downer'}

Nearly $60 \%$ of the participants reported that the strongest 'downer' when meeting a person for the first time was sweat malodour (59.2\%). For 37.2\% this appeared to be breath malodour, for $2.8 \%$ dishevelled appearance due to facial, ear or nasal hair, and for $0.8 \%$ dirty ears. Participants older than 40 years were significantly more likely than participants younger than 40 years to report halitosis as being the strongest 'downer' (40.5\% vs 31.4\%, chi-square test [3]: 9.6; $p=0.022$ ). When the groups of participants with the two lowest levels of education were combined, it appeared that they were significantly less likely to consider halitosis as the strongest 'downer' than the participants with the highest level of education $(35.0 \%$ vs $43.2 \%$, chi-square test [1] $=5.7 ; p=0.017)$.

\section{Drawing attention to a person's halitosis}

The answer to the question whether a participant would draw a person's attention to his breath malodour proved to be related to the kind of person involved (Fig. 1). Generally, the likelihood of drawing a person's attention to his breath malodour appeared to decrease with the increase of the social distance to the person. Regarding their spouses, 96.8\% (95\% CI: 95.7-98.0) of the participants responded positively. When a family member or friend would be involved, these percentages were $74.7 \%$ (95\% CI: 71.9-77.4) and 73.1\% (95\% CI: 70.3-75.8) respectively. Further, if the person would be a colleague, the boss or supervisor, or an unknown, accidentally met person 45.0\% (95\% CI: 41.8-48.2), 30.3\% (95\% CI: 27.3-33.3), and 7.9\% (95\% CI: 6.2 - 9.6) of the participants responded positively, respectively.

\section{Method of raising the problem of halitosis}

The participants indicated that drawing a person's attention to his breath malodour would be performed personally. In case of the spouse this was 95.5\% of the participants. With respect to a family member, a friend, a colleague, the boss or supervisor, and an unknown, accidentally met person, 75.9\%, $73.8 \%, 37.4 \%, 24.2 \%$ and $5.8 \%$ of the participants respectively would raise this problem personally. In case of a colleague, men more than women would raise the problem personally (44.5\% vs 29.6\%), whereas the likelihood that women would not raise the problem with a colleague at all was significantly higher than men $(51.7 \%$ vs 39.8\%; chi-square test [6]: 26.1; $p<0,001$ ). A similar gender difference was found with regard to raising the problem personally with the boss or supervisor (men 30.9\% vs women $16.6 \%$ ), whereas women were more likely than men to not raise the problem with their boss or supervisor at all (67.7\% vs 53.1\%; chi-square test [6]: 29.7; $\mathrm{p}<0.001$ ).

Indirect ways to raise the problem of halitosis were virtually not reported. It appeared that only $7.6 \%$ of the participants arranged for 'somebody else' to raise the problem. Other methods of drawing attention to a person's breath malodour, such as a note/e-mail (3.3\%), a sms/ping/whatsapp (1.3\%) or through a dentist or dental hygienist (3.3\%) were reported scarcely.

\section{DISCUSSION}

According to the best of our knowledge, this is the first study that investigates the impact of general population subjects faced with halitosis patients on their psychosocial interactions with these halitosis patients. Since the survey sample is representative for the general population aged 16 years and older in the Netherlands, the results indicated that almost $90 \%$ of the population of the Netherlands is facing people who suffer from halitosis regularly, $40 \%$ of them at least once 
Table 1 Comparison of demographic data of the study sample and the general population of the Netherlands aged 16 years and older

\begin{tabular}{|c|c|c|c|c|}
\hline Demographic variable & Sample no & Sample (\%) & $\begin{array}{l}\text { Population of the } \\
\text { Netherlands }(\%)\end{array}$ & $\begin{array}{l}\text { Outcome, chi- } \\
\text { squared test (\%) }\end{array}$ \\
\hline Gender & & & & (1): $0.402 ; p=0.526$ \\
\hline Female & 501 & 49.8 & 50.8 & \\
\hline Men & 505 & 50.2 & 49.2 & \\
\hline Age & & & & (4): $5.568 ; p=0.234$ \\
\hline $16-29$ & 183 & 18.2 & 21.1 & \\
\hline $30-39$ & 180 & 17.9 & 16.9 & \\
\hline $40-49$ & 201 & 20.1 & 19.4 & \\
\hline $50-59$ & 170 & 16.8 & 16.9 & \\
\hline$\geq 60$ & 272 & 27.0 & 25.7 & \\
\hline Family situation & & & & (2): $3.460 ; p=0.177$ \\
\hline Single & 184 & 18.3 & 20.1 & \\
\hline \multicolumn{5}{|l|}{ Cohabiting/married } \\
\hline (no children) & 496 & 49.3 & 46.6 & \\
\hline \multicolumn{5}{|l|}{ Cohabiting/married } \\
\hline (with children) & 326 & 32.4 & 33.3 & \\
\hline Education level & & & & (2): $2.638 ; p=0.267$ \\
\hline High school & 356 & 35.4 & 37.4 & \\
\hline College & 384 & 38.2 & 38.1 & \\
\hline Post graduate & 266 & 26.4 & 24.5 & \\
\hline Labour participation & & & & (2): $1.673 ; p=0.433$ \\
\hline Full-time & 366 & 36.4 & 34.7 & \\
\hline Part-time & 201 & 20.0 & 21.3 & \\
\hline No job & 439 & 43.6 & 44.0 & \\
\hline
\end{tabular}

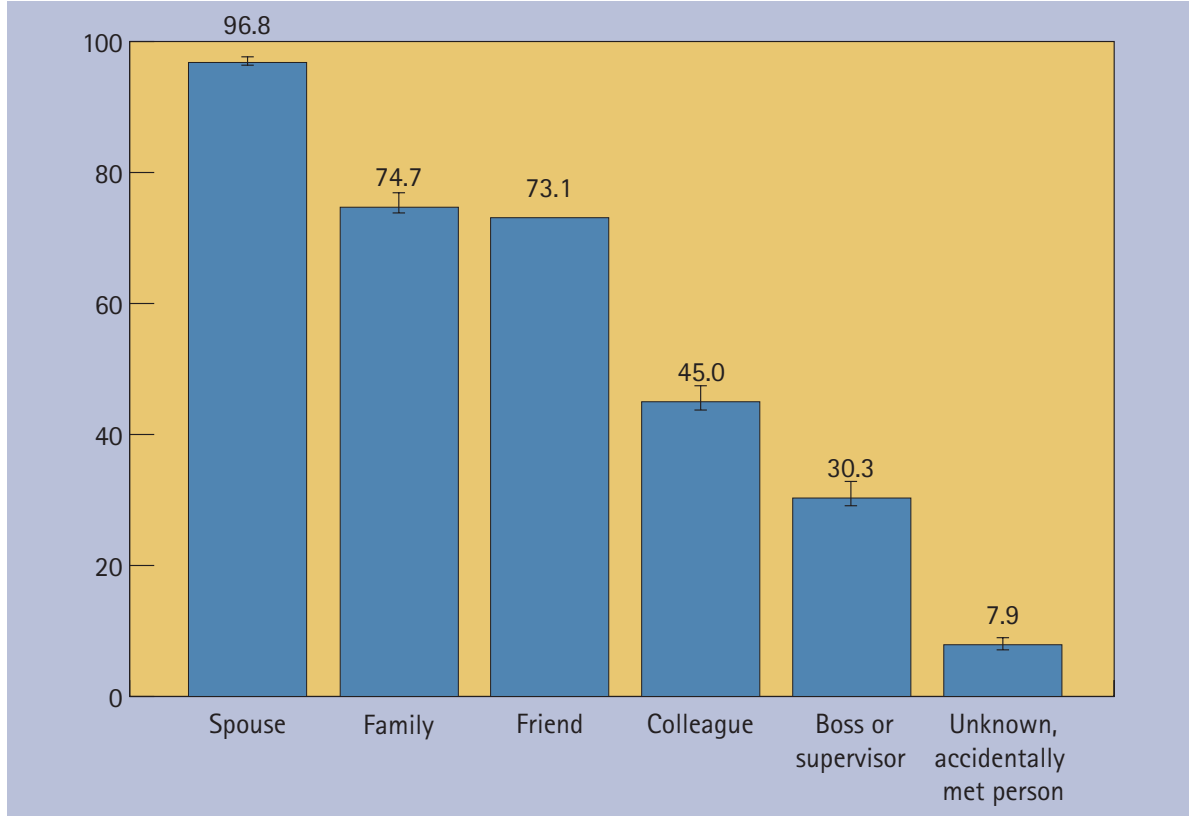

Fig. 1 Bar chart representing categories of persons in relation to likelihood (percentage) that one would draw this person's attention to his or her breath malodour (95\% confidence intervals are indicated). a week. The results further showed that halitosis is considered to be one of the most potentially unattractive aspects of social interactions. Probably the most significant finding of the present study was that the social distance to a person suffering from halitosis determines people's likelihood to draw this person's attention to his breath malodour.

Interestingly, significantly more men than women reported to be faced daily or weekly with a person having halitosis. One explanation for this gender difference might be that usually men take more notice of halitosis than women. However, this explanation is not very likely since the olfactory system of women is more sensitive than the olfactory system of men. ${ }^{15,16}$ It could also be argued that men are less reluctant than women to disclose personal information about their confrontations with a person's halitosis. However, studies of demographic differences in responses to sensitive survey items, such as depression, did not show such gender differences. ${ }^{17}$ Perhaps a more realistic explanation for this gender difference might be that men are faced more frequently with persons having halitosis, since men generally have a higher employment rate than women. Indeed, more participants with labour participation than those without labour participation indicated to be faced with halitosis regularly. Conceivably, social interactions during work demand a certain physical proximity, for instance during work consultations or contact with customers, which induces exposure to halitosis.

For relatively many participants being faced with a person's halitosis is a real 'downer'. Participants with a high education level and participants older than 40 years indicated breath malodour as the strongest 'downer' more frequently than those with a lower education level and those who were younger than 40 years. The latter finding is intriguing as one would imagine that younger individuals would have a higher level of concern for halitosis because of their desire for sexual or other close contact with their peers. It is also at odds with results of olfactory studies, which showed that over the age of 40 years olfactory acuity is more likely to decline (hyposmia) than to increase..$^{16}$ On the other hand, it is known that the ability to smell varies across age groups in odorant-specific fashion, and that people can develop a sensitivity for or a fear of scents during their life span. ${ }^{18,19}$ Whether this also applies to the volatile sulphur compounds involved in halitosis has, as far as we know, not been investigated yet. 
The results of the present study suggest the existence of a taboo with regard to drawing a person's attention to his or her halitosis, albeit depending on the social distance to the person. For instance, in case of a spouse the participants generally did not seem to experience difficulties in drawing attention. But when it comes to an unknown, accidentally met person, drawing attention to his or her halitosis personally would be done by less than $6 \%$ of the participants. Modern types of communication, albeit increasingly popular in social interactions nowadays, such as sending an e-mail or a text message, were indicated to be used seldom for this purpose. Although a certain level of reluctance to drawing a person's attention to his/her halitosis is certainly conceivable, it is also problematic, because the feelings of disgust that halitosis evokes in those who are faced with it could create social distance and alienate people from each other. Therefore, a relevant question in this respect is which effect is inflicted on a person when the issue of halitosis is addressed and he/she will become aware of the fact that other people notice his/her halitosis. Fear of insulting another person, making this person feeling uncertain or embarrassed, and the unpredictability of the consequences it might have for their relationship, could be an explanation for the finding that people are reluctant to alert a person about the presence of halitosis in case this person is not socially close or familiar to them.

The interpretation of the results should take into account some limitations. Given that there is almost no literature on the issue of unattractive aspects of social interactions, and to what extent these might be influenced by individuals' halitosis, the survey items were chosen on the basis of common sense and ideas from the research team. Accordingly, whether halitosis would have been ranked as an important factor alongside other potential options, such as facial disfigurement, facial tattooing and piercing or, for instance, urinary incontinence, remains unclear. Secondly, due to the sensitivity of the issue, the present study may have underestimated the number of participants who are willing or afraid to draw a person's attention to his halitosis because of effects of social desirability. However, bias caused by underestimation was expected to be a minor issue because of the use of an online survey rather than a face-to-face interview, which is a relatively anonymous method of self-reporting. ${ }^{20} \mathrm{An}$ additional advantage of a panel as used in the present study is that respondents selected are demographically balanced.

The results of the present study underline the importance of the potential long-lasting consequences of persistent halitosis in terms of its potential damaging effects on social interactions and relationships. Therefore, future research is needed to test accessible methods of drawing a person's attention to his/her halitosis, being the first step of seeking treatment. In this respect it would also be enlightening to investigate the extent to which there is a similar reluctance among dentists and dental hygienists to address the issue of halitosis. This potential reluctance may hamper an adequate treatment of the often offensive and debilitative problem of halitosis. Clearly, the present study is one of the first attempts to determine the impact of halitosis on psychosocial interactions. The results may generate hypotheses that inspire further investigation in this interdisciplinary area of oral biology, dentistry and social sciences.

\section{CONCLUSION}

Halitosis is a highly unattractive aspect of social interactions and may have longlasting consequences in terms of damaging effects on psychosocial interactions and relationships.

This work was funded by MedaPharma BV Netherlands.

1. van den Broek A M W T, Feenstra $L$, de Baat C. A review of the current literature on aetiology and measurement methods of halitosis. J Dent 2007; 35: $627-635$.
2. Hughes F J, McNab R. Oral malodour - a review. Arch Oral Biol 2008; 53 (Suppl. 1): S1-S7.

3. Scully C, Greenman J. Halitosis (breath odour). Periodontology 2000 2008; 48: 66-75.

4. Armstrong B L, Sensat M L, Stoltenberg J L. Halitosis: a review of current literature. J Dent Hyg 2010; 84: 65-74.

5. Scully C, Greenman J. Halitology (breath odour: aetiopathogenesis and management). Oral Dis 2012; 18: $333-345$.

6. Bosy A. Oral malodour: philosophical and practical aspects. J Can Dent Assoc 1997; 63: 196-201.

7. Oho T, Yoshida Y, Shimazaki Y, Yamashita Y, Koga T. Psychological condition of patients complaining of halitosis. J Dent 2001; 29: 31-33.

8. Quirynen $M$, Avontroodt $P$, Soers $C$, Zhao H, Pauwels $M$, Van Steenberghe D. Impact of tongue cleansers on microbial load and taste. J Clin Periodonto/ 2004 31: 506-510.

9. Suzuki $N$, Yoneda M, Naito T, Iwamoto T, Hirofuji T. Relationship between halitosis and psychological status. Oral Surg Oral Med Oral Pathol Oral Radiol Endod 2008; 106: 542-547.

10. Nardi G M, Forabosco A, Forabosco G, Musciotto A, Campisi G, Grandi T. Halitosis: a stomatological and psychological issue. Minerva Stomatol 2009; 58: 435-444.

11. Suzuki N, Yoneda M, Naito T et al. Association between oral malodour and psychological characteristics in subjects with neurotic tendencies complaining of halitosis. Int Dent J 2011; 61: 57-62.

12. Falcão D P, Vieira C N, De Amorim R F B. Breaking paradigmas: a new definition for halitosis in the context of pseudo-halitosis and halitophobia. J Breath Res 2012; 6: 017-105.

13. Zaitsu T, Ueno M, Shinada K, Wright F A, Kawaguchi Y. Social anxiety disorder in genuine halitosis patients. Health Qual Life Outcome 2011; 9: 94

14. Dutch Central Bureau of Statistics (CBS): Population statistics 2011 (in Dutch). (Cited December 6: 2012) Available at: URL: http: //statline.cbs.nl/StatWeb.

15. Doty R L, Appelbaum S, Zusho H, Settle R G. Sex differences in odour identification ability: a crosscultural analysis. Neuropsychologia 1985; 23: 667-672.

16. Hummel T, Kobal G, Gudziol H, Mackay-Sim A. Normative data for the "Sniffin Sticks" including tests of odour identification, odour discrimination, and olfactory thresholds: an upgrade based on a group of more than 3,000 subjects. Eur Arch Otorhinolaryngol 2007; 264: 237-243.

17. Aneshensel C S, Frerichs R R, Clark V A, Yokopenic $P A$. Measuring depression in the community: $A$ comparison of telephone and personal interviews. Public Opin Quart 1982; 46: 110-121.

18. Wysocki C J, Gilbert A N. National Geographic Smell Survey: effects of age are heterogenous. Ann NY Acad Sci 1989; 561: 12-28.

19. Takahashi L K, Nakashima B R, Hong H, Watanabe $K$. The smell of danger: a behavioural and neural analysis of predator odour-induced fear. Neurosci Biobehav Rev 2005; 29: 1157-1167.

20. Kissinger $P$, Rice J, Farley T et al. Application of computer-assisted interviews to sexual behaviou research. Am J Epidemiol 1999; 149: 950-954. 